\title{
SOME SPACES THAT DO NOT HAVE THE COMMON FIXED POINT PROPERTY
}

\author{
JOHN PHILIP HUNEKE ${ }^{1}$ AND HENRY H. GLOVER
}

\begin{abstract}
For what topological spaces $X$ do every pair of self maps of $X$ which commute under composition have a common fixed point? No nontrivial examples of such spaces are known. Since every self map commutes with itself, $X$ does not have this property if $X$ does not have the fixed point property. It is shown that every completely regular Hausdorff space containing an arc does not have this property. In general, the self maps for these spaces are not surjective. The image is the arc. For surjective self maps it is shown that every topological manifold with nonnegative Euler characteristic does not have this property. An earlier counterexample for the closed interval is used in all proofs. This counterexample is due to Huneke.
\end{abstract}

A topological space $X$ is said to have property $\mathrm{CFP}^{2}$ provided every two self maps which commute under composition have a common fixed point. In [4] Huneke shows that the closed unit interval, $I$, does not have $\mathrm{CFP}^{2}$. However if one of the two self maps of this interval is open, Joichi and Folkman (see [6]) show that commuting self maps of $I$ do have a common fixed point.

E. R. Fadell raised the question whether the fixed point property implies $\mathrm{CFP}^{2}$ for simply connected closed topological manifolds. A first negative answer to this question is given by Proposition 1 which says that every topological manifold $M$ does not have CFP ${ }^{2}$ by virtue of self maps $f, g$ which have the property that $f(M) \cup g(M) \subset A$, an $\operatorname{arc}$ in $M$.

As a result we study surjective maps. Let $X$ have property $\mathrm{CFP}^{2} \mathrm{~S}$ if $X$ has $\mathrm{CFP}^{2}$ for surjective self maps. The main result of this paper (Corollary 1 ) is that all topological manifolds $M$ with nonnegative Euler characteristic, do not have CFP'S. Huneke [5] first obtained this result for projective spaces over the real and complex fields.

A natural question is whether all manifolds $M$ fail to have CFP'S. For this it is sufficient to know that all manifolds with the fixed point property have positive Euler characteristic. This result is unknown to the writers.

Received by the editors June 16, 1969 and, in revised form, October 30, 1970. AMS 1970 subject classifications. Primary 55C20; Secondary 57A99.

Key words and phrases. Common fixed point, commuting under composition, topological manifolds.

${ }^{1}$ Supported in part by The National Science Foundation, GP-20871 through the University of Minnesota. 
Another question is whether $X$ has $\mathrm{CFP}^{2}$ for homeomorphisms. The answer is unknown to the writers even for the unit disk.

Our technique of construction is to find a surjective self map $f: M \rightarrow M$, homotopic to the identity map, and with fixed point set whose components have invariant and complement invariant neighborhoods (see Theorem 1). We then use the counterexample to $\mathrm{CFP}^{2}$ for the closed interval locally and in one coordinate to construct the desired maps.

Proposition A. Let $X$ be a completely regular Hausdorff space which contains an arc. Then there exist two continuous maps from $X$ onto the arc in $X$ which commute without common fixed points.

Proof. In [4] it is shown that there exist two self maps of $[-1,1]$, $f$ and $g$, which commute without common fixed points. Let $h$ be a homeomorphism from the arc in $X$ on to $[-1,1]$. Let $\bar{h}$ be Tietze's extension of $h$ to $\beta X$, the Stone-Čech compactification of $X$. Then $\left.h^{-1} \cdot f \cdot \bar{h}\right|_{x}$ and $\left.h^{-1} \cdot g \cdot \bar{h}\right|_{x}$ are self maps of $X$ which commute without common fixed points.

Notation. Throughout let $R^{m}$ denote $m$-dimensional euclidean space, $m>0$. Let $H^{m}=\left\{\left(x_{1}, \cdots, x_{m}\right) \in R^{m}: x_{m} \geqq 0\right\}$ denote $m$-dimensional euclidean half space. Let $M$ denote a topological manifold of dimension $m$, i.e., a Hausdorff space such that each point has an open neighborhood which is either homeomorphic to $R^{m}$ or else to $H^{m}$. If $x \in M$ has an open neighborhood homeomorphic to $R^{m}$ we say that $x \in$ interior $M$ (int $M$ ). If $x \notin$ int $M$ then we say that $x \in$ boundary $M$ (bd $M$ ).

If $M$ is compact and bd $M=\varnothing$ we say that $M$ is closed.

If $M$ is not compact we say that $M$ is open.

We have an immediate consequence of Proposition A.

Corollary A. Every topological manifold $M$ does not have CFP'.

Notation. Let $M$ be a topological manifold. Let $M_{0}$ denote the complement of a point interior to $M$. Let $\chi M$ denote the Euler characteristic of $M$.

LeMma 1. Every connected topological manifold $M$, with empty boundary, admits a surjective self map $f: M \rightarrow M$ such that

(1) $f$ is $\epsilon$-homotopic to the identity map.

(2) $f$ has at most one fixed point.

(3) $f$ is fixed point free if $M$ is open or $\chi M=0$.

Proof. The pair of maps $M \stackrel{\Delta}{\rightarrow} M \times M \stackrel{\mathrm{pr}_{1}}{\rightarrow} M$ defined by $\Delta(x)=(x, x)$, $\operatorname{pr}_{1}(x, y)=x$ is a basic example of a microbundle. Kister and Mazur 
(see [7]) have shown that every microbundle over a paracompact base space contains a fiber bundle with euclidean fiber. For this example this means that there exists an open neighborhood of $\Delta(M)$ in $M \times M, T M$, such that $\left.\mathrm{pr}_{1}\right|_{T M}$ is a fiber bundle with euclidean fiber. We call this bundle "the" tangent bundle of $M$ and let $T_{0} M$ denote $T M-\Delta(M)$. Since $\pi_{i}\left(R^{m}, R_{0}^{m}\right)=0$, if $i<m$, and $H^{m} M_{0}=0$ we may use obstruction theory to deform the section $\Delta$ into a section $s: M \rightarrow T M$ such that $s\left(M_{0}\right) \subset T_{0} M$. As a result $f=\mathrm{pr}_{2} \cdot s$ is a map with exactly one fixed point. Here $\operatorname{pr}_{2}(x, y)=y$. Let $d \in H^{m} M$ denote the obstruction to deforming $s$ to a section $s_{0}: M \rightarrow T M$ such that $s(M) \subset T_{0} M$. If $M$ is open, $H^{m} M=0$, and if $M$ is closed, $\mathrm{R}$. Brown [2] shows that $d=X$, the Euler class of $M$. Recall that $X=\chi M \cdot \mu$, where $\mu$ is the fundamental cohomology class of $M$. As a result $f=\mathrm{pr}_{2} \cdot s_{0}$ is the map desired in (3) above. Note that since $f$ is homotopic to the identity map it is surjective. Q.E.D.

LEMMA 2. Every topological manifold $M$ admits a surjective self map $f: M \rightarrow M$ such that

(1) $f$ is $\epsilon$-homotopic to the identity map.

(2) $f($ bd $M$ ) $\subset$ bd $M$.

(3) $f$ has at most one interior fixed point and has at most one fixed point on each component $C$, of bd $M$.

(4) $f$ is fixed point free on int $M$ if $M$ is open or $\chi M=0 . f$ is fixed point free on $C$, a component of bd $M$, if $\chi C=0$ or $C$ is open.

Proof. Let $f:$ bd $M \rightarrow$ bd $M$ be the map given on each component of bd $M$ by Lemma 1 . Morton Brown [1] has shown that bd $M$ has a neighborhood, $N$, homeomorphic to bd $M \times I$. As a result we can extend $f$ to int $N$ with the desired properties. Define $s: N \rightarrow N \times N$ by $s(x)=(x, f(x))$. Since $H^{m}\left(M_{0}\right.$, bd $\left.M\right)=0$, we may use obstruction theory to extend the deformation of the section $\Delta$ to $s$ :int $M \rightarrow T$ (int $M$ ) such that $s$ (int $M) \subset T_{0}$ (int $M_{0}$ ). As a result $f=\mathrm{pr}_{2} \cdot s$ is a surjective self map with properties (1)-(4).

Notation. Recall that $U \subset M$ is called invariant under $f: M \rightarrow M$ if $f(U) \subset U$. We say that $U \subset M$ is complement invariant under $f: M \rightarrow M$ if $f(M-U) \subset(M-U)$. Let $U \subset R^{m}$ be a euclidean neighborhood of the origin and let $f: U \rightarrow R^{m}$ be a map with exactly one fixed point at the origin. Recall that the index of this fixed point under $f$ is defined to be the degree of the difference map $\delta f: U_{0} \rightarrow R_{0}^{m}$ defined by $\delta f(x)$ $=f(x)-x$. Here $R_{0}^{m}$ and $U_{0}$ denote the complement of the origin in $R^{m}$ and $U$ respectively.

Let $r B^{m}=\left\{x \in R^{m}:|x| \leqq r\right\}$ denote the closed ball in $R^{m}$ of radius $r$. 
LEMMA 3. Let the origin be the only fixed point for $f: 3 B^{2 k} \rightarrow R^{2 k}$, and let $f\left(3 B^{2 k}-2 B^{2 k}\right) \cap B^{2 k}=\varnothing$. Suppose the index of the fixed point is 1 . Then there exists a map $g: 3 B^{2 k} \rightarrow R^{2 k}$ such that

(1) $\left.g\right|_{\left(3 B^{2 k}-2 B^{2 k}\right)}=\left.f\right|_{\left(3 B^{2 k}-2 B^{2 k}\right)}$.

(2) The origin is the only fixed point under $g$.

(3) $B^{2 k}$ is invariant and complement invariant under $g$.

(4) $\left.g\right|_{B^{2 k}}$ is given by $g(x)=-x$.

Proof. Note that there exists a homotopy equivalence between $B_{0}^{2 k}$ and $3 B^{2 k}-2 B^{2 k}$. Since the index of the fixed point under both $f$ and $g$ is $1,\left.s g\right|_{B_{0}^{2 k}}: B_{0}^{2 k} \rightarrow T_{0}^{2 k} R^{2 k}$ is homotopic to $\left.s f\right|_{\left(3 B^{2 k}-2 B^{2 k}\right)}: 3 B^{2 k}$ $-2 B^{2 k} \rightarrow T_{0} R^{2 k}$ under this equivalence. As a result there exists an

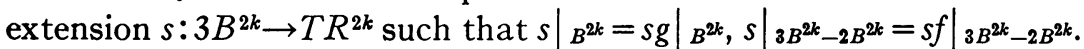
Define $g=\mathrm{pr}_{2} \cdot s$. This map has properties (1)-(4).

The proof of the following lemma is similar to the proof of Lemma 3.

Lemma 4. Let $(0,0),(0,-1),(0,1)$ be the only fixed points for $f: 3 B^{2 k} \times I \rightarrow R^{2 k} \times I$, and let $f\left(3 B^{2 k} \times I-2 B^{2 k} \times I\right) \cap B^{2 k} \times I=\varnothing$. Suppose that $f\left(3 B^{2 k}, t\right) \subset\left(R^{2 k}, t\right), t= \pm 1$, and suppose the index of each of these fixed points is 1 . Then there exists a map $g: 3 B^{2 k} \times I \rightarrow R^{2 k} \times I$ such that

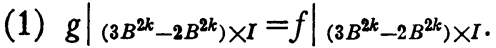

(2) $B^{2 k} \times I$ is invariant and complement invariant under $g$.

(3) $\{0\} \times I$ is the fixed point set of $g$.

(4) $\left.g\right|_{B^{2 k} \times I}$ is given by $g(x, t)=(-x, t)$.

Notation. Let $x_{0} \in$ int $M$ be an isolated fixed point for the map $f: M \rightarrow M$. Let $U \subset V \subset M$ be a pair of euclidean neighborhoods of $x_{0}$ such that $f(U) \subset V$ and $x_{0}$ is the only fixed point for $\left.f\right|_{U}$. We define the index of $x_{0}$ under $f$ to be the index of $\left.f\right|_{U}$.

REMARK. The index of the isolated fixed point $x_{0} \in$ int $M$ for $f: M \rightarrow M$ is well defined, i.e., the index does not depend on the choice of the pair of euclidean neighborhoods $U \subset V \subset M$.

TheOREM 1. Suppose $\chi M \geqq 0$. Then there exists a surjective self map $\phi: M \rightarrow M$ whose fixed point set has components $F_{i}$, with closed euclidean neighborhoods $U_{i}$ given by homeomorphisms

$$
\begin{array}{ll}
h_{i}: B^{2 k} \rightarrow U_{i}, & \text { if } m=2 k, \\
h_{i}: B^{2 k} \times I \rightarrow U_{i}, & \text { if } m=2 k+1,
\end{array}
$$

$i=1, \cdots, \chi M$, such that

(1) $U_{i}$ is invariant and complement invariant for all $i$.

(2) $U_{i} \cap U_{j}=\varnothing$ unless $i=j$. 
(3) $h_{i}^{-1} \cdot \phi \cdot h_{i}: B^{2 k} \rightarrow B^{2 k}$, is given by $x \mapsto-x$, if $m=2 k$, and $h_{i}^{-1} \cdot \phi$ $\cdot h_{i}: B^{2 k} \times I \rightarrow B^{2 k} \times I$ is given by $(x, t) \mapsto(-x, t)$, if $m=2 k+1$, for all $i$.

(4) $h_{i}(x, t) \in$ bd $M$ if $t= \pm 1$ and $m=2 k+1$, for all $x$ and $i$.

Proof. Case (i). bd $M=\varnothing$. If $M$ is open apply Lemma 2 . If $M$ is closed and $m=2 k+1$ we conclude by Poincaré duality that $\chi M=0$ and apply Lemma 2 . If $M$ is closed and $m=2 k$ we apply Lemma 2 to get a map $f_{0}: M \rightarrow M$ with at most one fixed point $x_{0}$. R. Brown [2] shows that $f_{0}$ is $\epsilon$-homotopic to a map $f_{1}$ with $\chi M$ fixed points $x_{0}^{i}$, each of index 1 , which are sufficiently far apart with respect to $\epsilon$. This means that each fixed point $x_{0}^{i}$ has a euclidean neighborhood $U_{i}$ given by a homeomorphism $h_{i}: R^{2 k} \rightarrow U_{i}$ such that (1) $h_{i}(0)=x_{0}^{i}$, (2) $U_{i} \cap U_{j}$ $=\varnothing$, (3) $f_{1} \cdot h_{i}\left(3 B^{2 k}\right) \subset U_{i}$, (4) $f_{1}\left(M-h_{i}\left(2 B^{2 k}\right)\right) \cap h_{i}\left(B^{2 k}\right)=\varnothing$. Observe that $h_{i}^{-1} \cdot f_{1} \cdot h_{i}: 3 B^{2 k} \rightarrow R^{2 k}$ satisfies the hypotheses of Lemma 3. As a result

$$
\begin{aligned}
\phi(x) & =f_{1}(x), \quad x \in M-\bigcup_{i=1}^{\chi M} U_{i}, \\
& =h_{i} \cdot g_{i} \cdot h_{i}^{-1}(x), \quad x \in U_{i}, i=1, \cdots, \chi M,
\end{aligned}
$$

satisfies properties (1)-(4) where $g_{i}: 3 B^{2 k} \rightarrow R^{2 k}$ is given by Lemma 3 . Case (ii). bd $M \neq \varnothing$. Let $f_{0}: M \rightarrow M$ be the map given by Lemma 2 . If $m=2 k$ then $\operatorname{dim}$ bd $M=2 k-1$ and $f_{0}$ is fixed point free on bd $M$ by Lemma 2. If $M$ is open then we may apply Lemma 2. If $M$ is compact let $D f_{0}: D M \rightarrow D M$ be the induced map on the double of $M$. Since $\chi D M=2 \chi M-\chi$ bd $M=2 \chi M$ we may proceed as in Case (i). It remains to consider the case bd $M \neq \varnothing, M$ compact, $m=2 k+1$. Since $0=\chi D M=2 \chi M-\chi \mathrm{bd} M \mathrm{R}$. Brown [2] says that $f_{0}$ is $\epsilon$-homotopic to a map $f_{1}$ with $\chi M$ interior fixed points $x_{0}^{i}, i=1,2, \cdots, \chi M$ and $2 \chi M$ boundary fixed points $x_{-1}^{i}, x_{1}^{i}, i=1,2, \cdots, \chi M$ each of index 1 , which are sufficiently far apart with respect to $\epsilon$. The map $f_{1}$ also has the property that $f_{1}($ bd $M) \subset$ bd $M$. This means that each fixed point set $\left\{x_{0}^{i}, x_{-1}^{i}, x_{1}^{i}\right\}, i=1,2, \cdots, \chi M$, has a euclidean neighborhood $U_{i}$ given by a homeomorphism $h_{i}: R^{2 k} \times I \rightarrow U_{i}, i=1,2, \cdots, \chi M$, such that (1) $h_{i}(0,0)=x_{0}^{i}, h_{i}(0, \epsilon)=x_{i}, \epsilon= \pm 1$, are the only fixed points of $f \mid U_{i}$, (2) $U_{i} \cap U_{j}=\varnothing$, unless $i=j$, (3) $h_{i}\left(R^{2 k}, t\right) \subset$ bd $M$, if $t= \pm 1$, (4) $f_{1} \cdot h_{i}\left(3 B^{2 k} \times I\right) \subset U_{i}$, (5) $f_{1}\left(M-h_{i}\left(2 B^{2 k} \times I\right)\right) \cap h_{i}\left(B^{2 k}\right)=\varnothing$. We may now apply Lemma 4 to complete the proof of the theorem.

Corollary 1. If $\chi M \geqq 0$ then $M$ does not have $C F P^{2} S$.

Proof. In [4] it is shown that there exist self maps $f$ and $g$ of $[-1,1]$ which commute without common fixed points and which interchange -1 and 1 . 
Define $f_{1}, g_{1}: B^{2 k} \rightarrow B^{2 k}$ by

$$
f_{1}\left(x_{1}, x_{2}, \cdots, x_{2 k}\right)=\left(\alpha f\left(x_{1} / \alpha\right),-x_{2}, \cdots,-x_{2 k}\right)
$$

and

$$
g_{1}\left(x_{1}, x_{2}, \cdots, x_{2 k}\right)=\left(\alpha g\left(x_{1} / \alpha\right),-x_{2}, \cdots,-x_{2 k}\right),
$$

where $\alpha=\left(1-\sum_{i=2}^{2 k} x_{i}^{2}\right)^{1 / 2}$ and it is understood that $\alpha=0$ implies $\alpha f\left(x_{1} / \alpha\right)=0=\alpha g\left(x_{1} / \alpha\right)$. Observe that $\left.f_{1}\right|_{\text {bd } B^{2 k}}(x)=\left.g_{1}\right|_{\text {bd } B^{2 k}}(x)=-x$. Define $\bar{f}_{1}, \bar{g}_{1}: B^{2 k} \times I \rightarrow B^{2 k} \times I$ by $\bar{f}_{1}(x, t)=\left(f_{1}(x), t\right), \bar{g}_{1}(x, t)=\left(g_{1}(x), t\right)$. Let $\phi, h_{i}$, and $U_{i}$ be the maps and sets given by Theorem 1. Define $F$ and $G$, self maps of $M$, by:

$$
\begin{aligned}
F(x) & =\phi(x), & & \text { if } x \in M-\bigcup_{i=1}^{x^{M}} U_{i}, \\
& =h_{i} \cdot f_{1} \cdot h_{i}^{-1}(x), & & \text { if } x \in U_{i}, \quad m=2 k, \\
& =h_{i} \cdot \bar{f}_{1} \cdot h_{i}^{-1}(x), & & \text { if } x \in U_{i}, \quad m=2 k+1,
\end{aligned}
$$

and

$$
\begin{aligned}
& G(x)=\phi(x), \quad \text { if } x \in M-\bigcup_{i=1}^{x M} U_{i}, \\
& =h_{i} \cdot g_{1} \cdot h_{i}^{-1}(x), \quad \text { if } x \in U_{i}, \quad m=2 k, \\
& =h_{i} \cdot \bar{g}_{1} \cdot h_{i}^{-1}(x), \quad \text { if } x \in U_{i}, \quad m=2 k+1 \text {. }
\end{aligned}
$$

Maps $F$ and $G$ have the desired properties.

Corollary 2. Projective spaces over the real, complex, and quaternion fields do not have $C F P^{2} S$.

Proof. Projective spaces of odd dimension over the real and complex fields do not have the fixed point property so do not have CFP'S. The other projective spaces do have this property (see [3, pp. 18 , 22 ]), but also have positive Euler characteristic, so apply Corollary 1.

\section{REFERENCES}

1. Morton Brown, Locally flat imbeddings, Ann. of Math. (2) 75 (1962), 331-341. MR 24 \#A3637.

2. R. F. Brown, Path fields on manifolds, Trans. Amer. Math. Soc. 118 (1965), 180-191. MR $30 \# 3482$.

3. Edward Fadell, Recent results in fixed point theory of continuous maps, Bull. Amer. Math. Soc. 76 (1970), 10-29.

4. J. P. Huneke, On common fixed points of commuting continuous functions on an interval, Trans. Amer. Math. Soc. 139 (1969), 371-381. MR 38 \#6005. 
5. - On commuting functions without common fixed points, Notices Amer. Math. Soc. 16 (1969), 789-790. Abstract \#667-116.

6. J. T. Joichi, On functions that commute with full functions and common fixed points, Nieuw Arch. Wisk. 14 (1966), 247-251. MR 34 \#5078.

7. J. M. Kister, Microbundles are fibre bundles, Bull. Amer. Math. Soc. 69 (1963), 854-857. MR 27 \#6283.

The Ohio State University, Columbus, Ohio 43210 